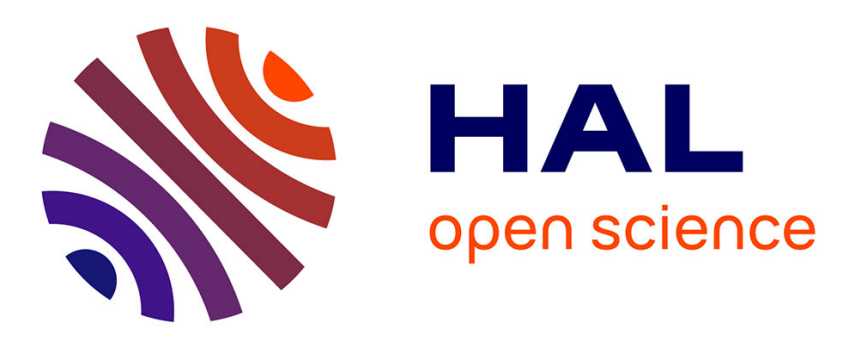

\title{
Automatic detection of well sampled images via a new ringing measure
}

\author{
Gwendoline Blanchet, Lionel Moisan, Bernard Rougé
}

\section{To cite this version:}

Gwendoline Blanchet, Lionel Moisan, Bernard Rougé. Automatic detection of well sampled images via a new ringing measure. 2010 IEEE International Conference on Acoustics Speech and Signal Processing (ICASSP), 2010, Dallas, United States. pp.1030 - 1033, 10.1109/ICASSP.2010.5495324 . hal-00388032v2

\section{HAL Id: hal-00388032 https://hal.science/hal-00388032v2}

Submitted on 7 Jan 2010

HAL is a multi-disciplinary open access archive for the deposit and dissemination of scientific research documents, whether they are published or not. The documents may come from teaching and research institutions in France or abroad, or from public or private research centers.
L'archive ouverte pluridisciplinaire HAL, est destinée au dépôt et à la diffusion de documents scientifiques de niveau recherche, publiés ou non, émanant des établissements d'enseignement et de recherche français ou étrangers, des laboratoires publics ou privés. 


\title{
AUTOMATIC DETECTION OF WELL SAMPLED IMAGES VIA A NEW RINGING MEASURE
}

\author{
Gwendoline Blanchet ${ }^{1}$, Lionel Moisan ${ }^{2}$, Bernard Rougé ${ }^{3}$ \\ ${ }^{1}$ Centre National d'Etudes Spatiales, Toulouse, France \\ ${ }^{2}$ Université Paris Descartes, MAP5, CNRS UMR 8145, Paris, France \\ ${ }^{3}$ École Normale Supérieure de Cachan, CMLA, CNRS UMR 8536, Cachan, France
}

\begin{abstract}
According to Shannon Sampling Theory, Fourier interpolation is the optimal way to reach subpixel accuracy from a properly-sampled digital image. However, for most images this interpolation tends to produce an artifact called ringing, that consists in undesirable oscillations near objects contours. In this work, we propose a way to detect this ringing artifact. Using Euler zigzag numbers, we compute the probability that neighboring gray-levels form an alternating sequence by chance, and characterize these undesirable ringing blocks as structures that would be very unlikely in a random image. We then show two applications where the associated algorithm is used to test or enforce the compliance of an image with Fourier interpolation.
\end{abstract}

Index Terms - Image sampling, ringing, Fourier interpolation, a-contrario detection.

\section{INTRODUCTION}

Inadequate sampling or processing of a digital image may cause various artifacts, including blur, aliasing, blocking, ringing, etc. Among them, the ringing artifact can be characterized by the presence of undesirable oscillations of the image intensities, generally located near objects contours. In this paper, we propose a new method to detect image zones containing high-frequency ringing, that is, oscillations at Nyquist frequency (period of 2 pixels), that may appear in particular when an image is interpolated in Fourier domain (the so-called "ideal sinc interpolation" in a periodic setting, see [1], Equation 26). Image down-scaling for example, can be performed optimally (in the $L^{2}$ sense) by band-limiting an image with a hard cutoff in the frequency domain prior to downsampling. However, this process generally causes ringing artifacts in the shrinked image, since the multiplication with a window function in the frequency domain is equivalent in the image domain to the convolution with an oscillating sinc kernel. High-frequency ringing also appears when an aliased image (that is, an image that has not been sampled above Nyquist rate) is interpolated in Fourier domain, and, as we shall see later, this phenomenon can be used on purpose to detect aliasing. More generally, as soon as subpixel interpolation is required for an image, ringing detection is a very interesting tool not only to decide whether the optimal Fourier interpolation can be used or not, but also to choose an appropriate image preprocessing filter or to select an alternative interpolation kernel.

All no-reference ringing metrics proposed in the literature are concerned by the presence of visible ringing in images, in particular as a compression artifact. Oguz [2] defines the Visible Ringing Measure after an edge map obtained from morphological processing. It has been recently used by Yang et al. [3] to assess the efficiency of a ringing removal algorithm for JPEG2000 images. Liu et al. [4] use a perceptually more relevant edge detector, but their ringing metric still depends on image structures (edges) and contrast requirements. Sheikh et al. [5] propose to learn natural scene statistics to rate the quality of JPEG2000 images. In all cases, parameters must be learned or tuned to mimic human perception.

In this work, we propose a way to detect high-frequency ringing in an image in a generic way by characterizing suspiciously large oscillating patterns with a probabilistic model. In Section 2, we obtain a criterion that allows the detection of ringing artifacts in a contrast-invariant way, while offering the guarantee that no detection occurs in a white noise image (whatever the noise distribution is). The resulting algorithm (Section 3) is then applied in Section 4 to test the compliance of an image with Fourier interpolation, and later in Section 5 to perform optimal ringing-free image down-scaling.

\section{A-CONTRARIO RINGING DETECTION}

By definition, high-frequency ringing results in alternated gray levels on a given line (see for example the right image of Fig. 3). If $g_{1}, g_{2}, \ldots g_{n}$ are the successive gray levels encountered on such a line, we shall say that they form an alternating sequence if

$$
\forall k \in\{1, . . n-2\}, \quad\left(g_{k+2}-g_{k+1}\right) \cdot\left(g_{k+1}-g_{k}\right)<0 .
$$

In other terms, the signs of $g_{2}-g_{1}, g_{3}-g_{2}, \ldots g_{n}-g_{n-1}$ are alternating like,,,$+-+ \ldots$ or,,,$-+- \ldots$ To decide whether an alternating sequence should be considered as normal or suspicious in an image, we propose to use the a-contrario framework [6] 
and compute the probability to obtain, by chance, an alternating sequence with length at least $n$. If the gray-levels $\left(g_{k}\right)$ are obtained from distinct pixels of a white noise image - that is, a random image $U$ such that all $U(x, y)$ are independent and identically distributed (i.i.d.) - , then each sign $g_{k+1}-g_{k}$ has a probability $1 / 2$ to be positive, and a probability $1 / 2$ to be negative. However, the successive signs are not independent, as shown by the following theorem.

Theorem 1 Let $U$ be a discrete white noise image, that is, i.i.d. gray values drawn according to a given probability measure admitting a density with respect to Lebesgue measure. Then, for any sequence of $n \geq 3$ distinct pixels, the probability that the corresponding $n$ gray levels form an alternating pattern is

$$
p_{n}=\frac{2 A_{n}}{n !},
$$

where $A_{n}$ is the $n$-th Euler zigzag number, that can be obtained from the exponential generating function

$$
\tan \left(\frac{x}{2}+\frac{\pi}{4}\right)=\tan x+\sec x=\sum_{n \geq 0} A_{n} \frac{x^{n}}{n !} .
$$

From classical expansions of $A_{n}$ and $n$ !, it is not difficult to show that

$$
p_{n} \underset{n \rightarrow \infty}{\sim} \tilde{p}_{n}, \text { with } \tilde{p}_{n}=4 \cdot\left(\frac{2}{\pi}\right)^{n+1},
$$

and the approximation is very good even for small $n$ (see Table 1). If the successive signs were independent, the probability to obtain an alternating pattern would be $q_{n}=2^{2-n}$, which asymptotically underestimates the actual probability $p_{n}$ by the non-negligible factor $(4 / \pi)^{n} \simeq 1.273^{n}$.

Now we come to the definition of an horizontal ringing block. In the following, $u$ is a gray-level image, that is a realvalued function defined on $\Omega=\{0, . . M-1\} \times\{0, . . N-1\}$. A rectangle of $\Omega$ is a subset $R=I \times J$ of $\Omega$, where $I$ and $J$ are intervals of $\mathbb{Z}$ (that is, sets made of consecutive integers). We shall say that $R$ has size $l \times w$ (length $l$, width $w$ ) if $|I|=l$ and $|J|=w$

\begin{tabular}{|c|c|c|c|c|}
\hline$n$ & $A_{n}$ & $n !$ & $p_{n}$ & $\left|\frac{\tilde{p}_{n}}{p_{n}}-1\right|$ \\
\hline 3 & 2 & 6 & 0.667 & $1.45 \cdot 10^{-2}$ \\
\hline 4 & 5 & 24 & 0.417 & $3.86 \cdot 10^{-3}$ \\
\hline 5 & 16 & 120 & 0.267 & $1.44 \cdot 10^{-3}$ \\
\hline 10 & 50521 & 3628800 & 0.0278 & $5.62 \cdot 10^{-6}$ \\
\hline 20 & $3.7 \cdot 10^{14}$ & $2.4 \cdot 10^{18}$ & $3.04 \cdot 10^{-4}$ & $9.56 \cdot 10^{-11}$ \\
\hline
\end{tabular}

Table 1. Euler zigzag numbers $\left(A_{n}\right)$ and $n$ ! permit to compute the probability $p_{n}$ that a sequence of gray values alternates in a white noise image (see Theorem 1). Note on the right column that the approximation of $p_{n}$ by its asymptotics $\tilde{p}_{n}$ becomes rapidly excellent as $n$ increases.
Definition 1 Le $u: \Omega \rightarrow \mathbb{R}$ be a gray-level image. A discrete rectangle $R=I \times J$ of $\Omega$ is a horizontal ringing block (HRB) if $(u(x, y))_{x \in I}$ is an alternating sequence for all $y \in J$.

Fig. 1 shows an example of a horizontal ringing block (HRB).

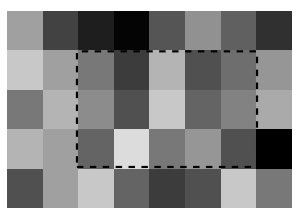

Fig. 1. Example of a $5 \times 3 \mathrm{HRB}$ (dashed rectangle) in a $8 \times 5$ image.

Thanks to Theorem 1 , the probability of a $l \times w$ rectangle to be a HRB in a random image is $\left(p_{l}\right)^{w}$. Let us consider the domain $D_{\infty}=\{3, . .+\infty\} \times\{1, . .+\infty\}$ and for any $\alpha>0$, the sub-domain $D_{\alpha}=\left\{(l, w) \in D_{\infty},\left(p_{l}\right)^{w} \leq \alpha\right\}$. We call $n(\alpha)$ the minimum number of discrete regions (quarterplanes) of the kind $\left\{l_{0}, . .+\infty\right\} \times\left\{w_{0}, . .+\infty\right\}\left(l_{0}\right.$ and $w_{0}$ being arbitrary integers) needed to cover exactly $D_{\alpha}$. Considering the shape of $D_{\alpha}$ (see Fig. 2), it is easy to show that $n(\alpha)$ is the number of horizontal (or vertical) lines required to draw the boundary of $D_{\alpha}$. The function $\alpha \mapsto n(\alpha)$ is nonincreasing, and since $p_{3}=2 / 3, n(\alpha)$ is bounded from above by $\left\lceil\frac{\log \alpha}{\log (2 / 3)}\right\rceil$ (upper integer part). Typical values of $n(\alpha)$ are $n\left(10^{-6}\right)=10, n\left(10^{-8}\right)=11, n\left(10^{-10}\right)=13$, $n\left(10^{-23}\right)=20$.

Definition 2 Let $u: \Omega \rightarrow \mathbb{R}$ be a $M \times N$ gray-level image. If $R$ is a $l \times w H R B$ of $u$, we write $\alpha(R)=M N \cdot\left(p_{l}\right)^{w}$, and say that $R$ is $\varepsilon$-meaningful if $\varphi(\alpha(R)) \leq \varepsilon$, where

$$
\varphi(\alpha)=\alpha \cdot n\left(\frac{\alpha}{M N}\right) .
$$

We say that $R$ is maximal $\varepsilon$-meaningful if there is no $\varepsilon$ meaningful HRB of $u$ that contains strictly $R$.

We expect that a ringing-free image will not contain $\varepsilon$ meaningful HRBs for $\varepsilon \ll 1$, since, as we shall see now, the expected number of maximal $\varepsilon$-meaningful HRBs in a random image is less than $\varepsilon$ (a-contrario property, see [6]).

Theorem 2 For any $\varepsilon>0$ and any $M \times N$ image $u$, let us call $m_{\varepsilon}(u)$ the number of maximal $\varepsilon$-meaningful HRBs of $u$. Then for any white noise image $U$, one has

$$
\forall \varepsilon>0, \quad \mathbb{E}\left(m_{\varepsilon}(U)\right) \leq \varepsilon .
$$

Proof - Any maximal $\varepsilon$-meaningful HRB $R$ of $U$ satisfy $\alpha(R) \leq \bar{\alpha}$, where $\bar{\alpha}$ is the maximum value of $\alpha$ such that $\varphi(\alpha) \leq \varepsilon$. By definition of $n(\bar{\alpha})$, there exists a finite subset $C_{\bar{\alpha}}$ of $D_{\bar{\alpha}}$ such that

$$
D_{\bar{\alpha}}=\bigcup_{\left(l_{0}, w_{0}\right) \in C_{\bar{\alpha}}}\left\{l_{0}, . .+\infty\right\} \times\left\{w_{0}, . .+\infty\right\} .
$$




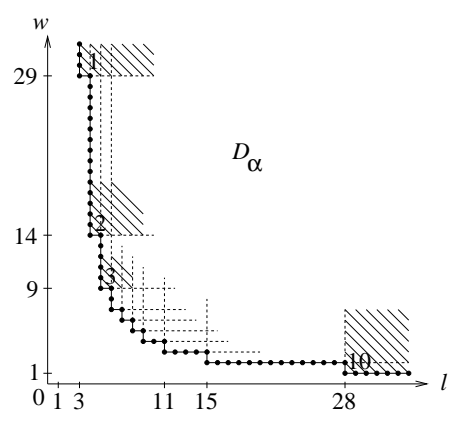

Fig. 2. The $D_{\alpha}$ domain for $\alpha=10^{-5}$ can be covered by 10 quarterplanes, that is, $n(\alpha)=10$.

Let us call minimal $\varepsilon$-meaningful HRB of $U$ any $l \times w \mathrm{HRB}$ of $U$ such that $(l, w) \in C_{\bar{\alpha}}$. By definition of $C_{\bar{\alpha}}$, any $\varepsilon$ meaningful HRB of $U$ (maximal or not) can be written as the union of minimal $\varepsilon$-meaningful HRBs of $u$. Hence, it is possible to build the set of maximal $\varepsilon$-meaningful HRBs of $U$ from the set of minimal $\varepsilon$-meaningful HRBs of $U$, by applying a recursive fusion process, and the number of maximal HRBs obtained this way will be smaller than the number of original minimal HRBs. Now, since the total number of possible minimal $\varepsilon$-meaningful HRBs is $M N \cdot n\left(\frac{\bar{\alpha}}{M N}\right)$ and the probability of one of these rectangles to be a HRB is less than $\frac{\bar{\alpha}}{M N}$, the expected number of minimal $\varepsilon$-meaningful HRBs is less than

$$
M N \cdot n\left(\frac{\bar{\alpha}}{M N}\right) \cdot \frac{\bar{\alpha}}{M N}=\varphi(\bar{\alpha}) \leq \varepsilon
$$

which concludes the proof.

\section{ALGORITHM}

Thanks to the previous results, we can tell if ringing is present in a $M \times N$ image $u$ using the following procedure:

1. Choose a bound $\varepsilon$ on the expected number of false alarms, for example $\varepsilon=0.01$ (we allow, in average, one false alarm every 100 images);

2. Find $\bar{\alpha}$ such that $\varphi(\bar{\alpha}) \leq \varepsilon$ (if $M=N=1000$ and $\varepsilon=0.01$, then $\bar{\alpha}=1 / 1100$ is convenient);

3. For each pixel $(x, y)$ of $u$, detect if it is a horizontal extrema, that is $u(x-1, y), u(x, y), u(x+1, y)$ form an alternating sequence;

4. Among these points, find all maximal rectangles, and dilate them by one pixel horizontally;

5. Among these rectangles, select maximal $\varepsilon$-meaningful HRBs by checking the condition $\alpha(R) \leq \bar{\alpha}$.

Image $u$ has ringing if the number of these rectangles is significantly greater than $\varepsilon$ (if $\varepsilon=0.01$, one rectangle is enough). Note that this algorithm is contrast-invariant (that is, the result is unchanged if $u$ is changed into $g \circ u$, where $g: \mathbb{R} \rightarrow \mathbb{R}$ is an increasing contrast change), and that it only depends on one parameter (the choice of $\varepsilon$, that controls the expected number of false alarms). We checked that the number of maximal $\varepsilon$-meaningful HRBs on white noise images remains small (generally 0 or 1 for $\varepsilon=1$, almost always 0 for $\varepsilon=0.1$ ), as guaranteed by Theorem 2. Of course, the scope of this algorithm is not restricted to white noise images: as usually seen for a-contrario models, setting thresholds on noise images (in which there is no doubt that we should not detect any structure) yields a good control of false alarms on real-world images. In a sense, the method we propose here is a definition of ringing, and it would be interesting to lead psychovisual experiments to see how closely this model is linked to our visual perception.

\section{APPLICATION TO FOURIER INTERPOLATION}

As we mentioned before, Fourier interpolation is the optimal way to reach subpixel accuracy from a properly-sampled image. However, most real-world images are undersampled (hence aliased), because undersampling yields a better appearance (it makes images look artificially sharper). A good way to check if an image has been well sampled is to resample it on a translated grid (typically by half of a pixel) and to see if ringing appears. Since we focused on horizontal ringing, this amounts to the following procedure :

1. Compute the periodic component $p$ of $u$ (see [1]);

2. Translate $p$ by $(1 / 2,0)$ in the Fourier domain, that is, compute the image $q$ whose Discrete Fourier Transform is $\hat{q}(a, b)=\hat{p}(a, b) \cdot \exp \left(\frac{-i \pi a}{M}\right)$;

3. Find all maximal 1-meaningful HRBs on $q$.

Step 1 permits to avoid ringing artifacts (edge effects) caused by the implicit periodization performed by the Discrete Fourier Transform (see [1]). It can be discarded but in that case, meaningful HRBs found near the image border have to be discarded too after Step 3.

We applied this procedure to the "caps" image taken from the Live database [7] (see Fig. 3). As expected, no ringing was found on the original image, whose quality is good. However, several ringing blocks were found on the translated image, which shows that this image is badly sampled, as Fourier interpolation creates ringing artifacts.

\section{APPLICATION TO IMAGE REDUCTION}

Thanks to Shannon Sampling Theorem, we know that if we want to down-scale an image to a given size without creating aliasing, the optimal way (in the $L^{2}$ sense) consists in changing its Fourier spectrum into a smaller one by cutting highfrequency components. Unfortunately, this hard frequency cut-off procedure has a drawback: it produces ringing (see Fig. 4, top row). Since the algorithm we proposed is able to detect ringing, we can try to perform ringing-free image reduction by selecting an adequate linear filter that will attenuate the high-frequency components of the shrinked image up 


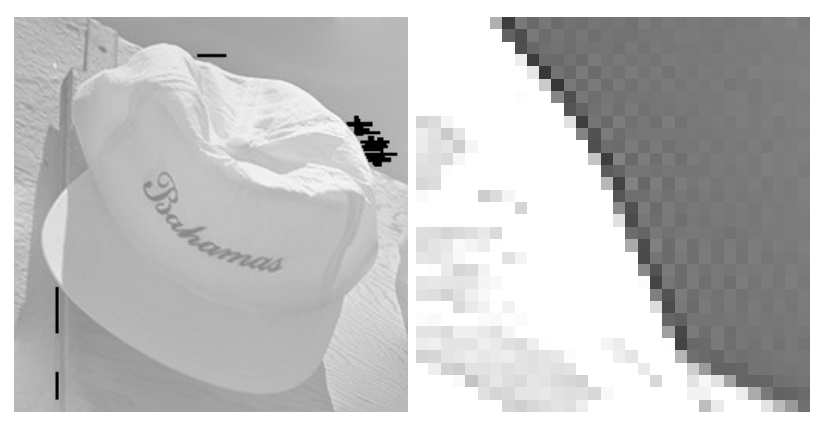

Fig. 3. The "caps" image from the Live database is not wellsampled: ringing is detected (black zones on the left image) after an horizontal translation of 0.5 pixel, especially near the top-right corner of the cap. The image on the right zooms on this part, with contrast magnifi cation and no detection marked.

to the point where the ringing artifacts disappear (but not further in order to introduce as little blur as possible). In practice, we considered a family of parametric filters $\left(h_{k}\right)$ and used the ringing detection to select the smallest value of the filter parameter $k$ yielding no ringing (see Fig. 4, bottom row). Compared to systematic lowpass filtering (see [8] in particular), the interest of this method is that it does not introduce a systematic amount of blur, but only the minimal necessary amount, so that the reduction of a blurry image may increase its perceived sharpness. Another interest is that the algorithm guarantees that the resulting image is well-sampled, which opens interesting perspectives concerning subpixel accuracy.

\section{CONCLUSION}

We proposed a new method to detect high-frequency ringing patterns in an image. As far as we know, this is the first attempt to detect ringing not only from a perceptual point of view (as done in [5] and [4]), but as an image processing nuisance (ringing patterns that are not perceived in an image can introduce strong artifacts when the image is processed, in particular if image derivatives are estimated). In fact, since the proposed algorithm is contrast-invariant, even poorly contrasted ringing patterns can be detected in an image provided that its SNR is large enough. Contrary to existing no-reference ringing metrics, no sensitive threshold has to be set and we have a statistical guarantee that the method we propose does not detect ringing in a random image. Despite its limitation to the mere detection of horizontal (or vertical) ringing patterns, our algorithm can be used to test the adequacy of an image with Fourier interpolation, or to reduce an image to the sharpest possible well-sampled one. Other applications involving high-frequency ringing artifacts could be investigated in a similar way, and the possible extension of the method to more general oscillating structures (lower frequencies, oblique directions, etc.) could lead to promising new applications (e.g., blind deconvolution with ringing control).

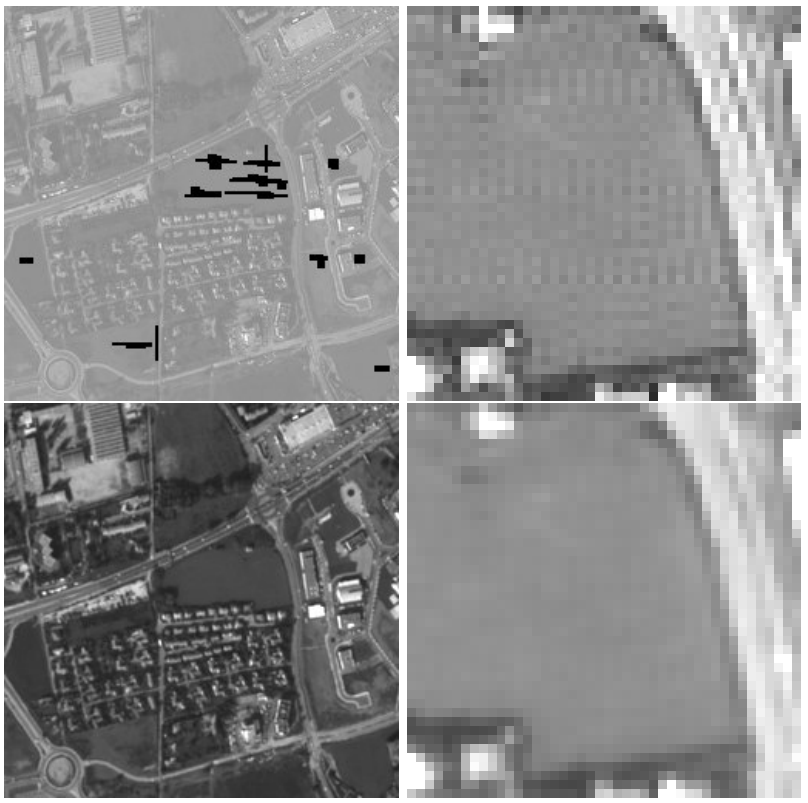

Fig. 4. The "nimes" image ( (C)CNES) is shrinked using a hard frequency cutoff (top left), which causes ringing artifacts (marked in black), visible on the magnifi ed part (top right). The ringing detector is then used to select an appropriate attenuation filter in the Fourier domain (cos fi lter), which result in a better-looking and ringing-free image (bottom row) that complies well with Fourier interpolation.

\section{REFERENCES}

[1] L. Moisan, 'Periodic plus smooth image decomposition," preprint 2009-11, MAP5, Université Paris Descartes, 2009.

[2] S.H. Oguz, Morphological post-filtering of ringing and lost data concealment in generalized lapped orthogonal transform based image and video coding, Ph.D. dissertation, Univ. of Wisconsin, Madison, 1999.

[3] S. Yang, Y. Hu, T. Nguyen, and D. Tull, 'Maximum-likelihood parameter estimation for image ringing-artifact removal," in IEEE Trans. on Circuits and Systems for Video Tech., 2001, vol. 11, pp. 963-973.

[4] H. Liu, N. Klomp, and I. Heynderickx, 'Perceptually relevant ringing region detection method," in proc. EUSIPCO, 2008.

[5] H.R. Sheikh, A.C. Bovik, and L. Cormack, 'No-reference quality assessment using natural scene statistics: Jpeg2000," IEEE Trans. Image Processing, vol. 14, no. 11, pp. 1918 - 1927, 2005.

[6] A. Desolneux, L. Moisan, and J.M. Morel, From Gestalt Theory to Image Analysis - A Probabilistic Approach, Springer, 2008.

[7] H.R. Sheikh, Z. Wang, L. Cormack, and A.C. Bovik, LIVE Image Quality Assessment Database Release 2, http://live.ece.utexas.edu/research/quality, 2005.

[8] G. Blanchet, L. Moisan, and B. Rougé, "A linear prefi lter for image sampling with ringing artifact control," in International Conference on Image Processing, 2005, vol. 3, pp. 577-580. 